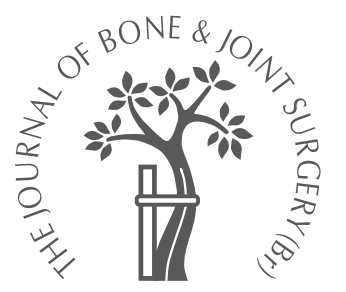

C. M. Robinson, R. J. Dobson

From the Royal

Infirmary of

Edinburgh,

Edinburgh, Scotland

C. M. Robinson, BMedSci, FRCS Ed (Orth), Senior Lecturer and Consultant Orthopaedic Surgeon

R. J. Dobson, Clinical

Research Assistant

The Shoulder Injury Clinic, Royal Infirmary of

Edinburgh, Old Dalkeith

Road, Little France,

Edinburgh EH16 4SU, UK.

Correspondence should be sent to $\mathrm{Mr}$ C. M. Robinson.

C2004 British Editorial

Society of Bone and

Joint Surgery

doi:10.1302/0301-620X.86B4.

$15014 \$ 2.00$

$J$ Bone Joint Surg [Br]

2004;86-B:469-79.

- REVIEW ARTICLE

\title{
Anterior instability of the shoulder after trauma
}

Anterior dislocation of the glenohumeral joint is a common injury of young people playing contact sport and may progress to recurrent episodes of symptomatic instability. The usual treatment for a primary dislocation is a short period of immobilisation followed by a programme of rehabilitation and a gradual return to full activity. An open operation to stabilise the shoulder is a successful procedure for the treatment of patients who develop recurrent instability. The advent of arthroscopic surgery, initially as a diagnostic tool, but more recently to perform therapeutic intervention, has resulted in a variety of minimally-invasive techniques for the management of instability.

Arthroscopic stabilisation done as day-care surgery offers attractive advantages over open repair such as improved cosmesis, less postoperative pain, reduced stiffness after operation and more rapid rehabilitation. As a consequence, this technique has been offered as initial treatment after a primary dislocation in an attempt to prevent subsequent instability. However, the results after this procedure have been much more variable than those for open repair.

In this review, we examine the recent advances in the understanding of the epidemiology and pathoanatomy of anterior instability of the shoulder after trauma, and compare the relative merits of arthroscopic and open stabilisation in the management of this condition.

\section{Who is at risk of shoulder instability?}

Primary anterior dislocation of the shoulder occurs commonly during contact sport in the young and after low-energy falls in the elderly. Although anterior dislocation in the aged has its own complications, ${ }^{1}$ recurrent instability is a particular problem in the young.

The typical patient with a high risk of developing recurrent instability is a man, either in his teenage years or early twenties, who suffers a primary dislocation while playing contact sports. The injury is usually sustained either with the arm in the 'at-risk' position of abduc- tion and external rotation, or from a direct impact to the shoulder. The symptoms of instability develop during the first two years after the original dislocation..$^{2-4}$

Despite the numerous studies of the natural history after an initial traumatic anterior dislocation, the exact incidence of recurrent instability remains uncertain, ${ }^{2,4,5}$ with rates quoted ranging from as low as $17 \%{ }^{6}$ to close to $100 \% .^{2,7-10}$ Many of the series which describe a high incidence have been drawn from selective populations, such as patients presenting to sports injury clinics and in military cadets,, 11 and may therefore be unrepresentative of the general population. There may also be a substantial pool of individuals who never seek medical advice, either because they sustain an isolated dislocation, become occasional dislocators, or are relatively asymptomatic and do not want surgical intervention. ${ }^{12}$

An accurate reflection of the true risk in unselected populations is probably an incidence of recurrent instability of $75 \%$ to $80 \%$ among individuals aged between 13 and 20 years and of $50 \%$ in those aged between 20 and 30 years. ${ }^{4,5,13}$ The high rate of recurrent instability during adolescence appears to be independent of whether the proximal humeral physis is open or not. ${ }^{9,10}$ The risk begins to decline in the late twenties, although recurrent instability is not uncommon in the middle-aged or in the elderly when it may be associated with significant problems of the rotator cuff. ${ }^{1}$

Although the age at the time of primary dislocation is the chief prognostic factor in determining the risk of recurrent instability, ${ }^{14}$ youth is also a marker for other important risk factors. These include an early return to competitive contact sports, poor compliance with rehabilitation and a greater likelihood of capsulolabral avulsion at the original dislocation. Many other factors have also been suggested as increasing the risk including a family history of recurrent instability, ${ }^{15}$ radiologically-visible avulsion fracture ${ }^{2,16}$ of the rim of the glenoid and a large Hill-Sachs lesion. ${ }^{4}$ 
Re-dislocation is rare if the greater tuberosity is fractured at the time of the initial injury, but these patients typically have a more protracted recovery, as a result of secondary dysfunction of the rotator cuff and impingement. ${ }^{17}$ The risk of re-dislocation may also be altered by the initial treatment.

\section{Definitions and classification}

Laxity of the shoulder, which is the normal translation of the humeral head over the surface of the glenoid, ${ }^{18}$ should be distinguished from instability, which occurs when the degree of translation becomes excessive and leads to symptoms. Instability may vary in its degree (subluxation, dislocation and micro-instability), direction (anterior, inferior, posterior and multidirectional), aetiology (traumatic or atraumatic) and volition (voluntary or involuntary).

Two separate groups of individuals who develop glenohumeral instability can be described $;^{19}$ namely, those who injure their shoulder (TUBS or traumatic, typically unilateral, with a Bankart lesion and usually requiring surgery to stabilise the shoulder) and those in whom instability develops insidiously as a result of an inherited constitutional ligamentous laxity (AMBRI or atraumatic, multidirectional, commonly bilateral, treatment by rehabilitation and inferior capsular shift in some refractory patients). Many of those in the latter group also have evidence of ligamentous laxity in other joints. Increasingly, these two acronyms are viewed as the ends of a spectrum of disease in which both traumatic and atraumatic elements may be present. A degree of inherited predisposition to traumatic instability is suggested by its occurrence bilaterally in a quarter of all patients, who often have a family history of recurrent dislocation. ${ }^{15,20}$ Conversely, many athletes who have evidence of constitutional ligamentous laxity only develop symptoms of instability after injury, and have predominantly unidirectional anterior instability.

It is now recognised that participants in overhead sports and athletes who throw are susceptible to a wide variety of disorders of the shoulder, including impingement dysfunction of the rotator cuff and acquired instability, which may occur concurrently to varying degrees. ${ }^{21-23}$ The classic symptoms of instability may be absent in these individuals, but if not recognised, may lead to failure of treatment.

The final group of patients with unstable shoulders are those with voluntary instability. These individuals are normally considered to be able to dislocate their shoulders at will and should be treated non-operatively. ${ }^{24}$ Increasingly, this view is seen as oversimplistic since some individuals may develop an acquired instability after many repetitive voluntary dislocations, and others may have an involuntary posterior 'positional' element to their instability, which may respond to biofeedback or, more rarely, to surgery. ${ }^{25}$

Instability of the shoulder therefore encompasses a wide spectrum of conditions which can be distinguished by their aetiology, severity, constitutional factors and volition. For this reason there is no generally accepted all-inclusive system of classification and an individual or algorithmic approach to diagnosis and treatment is probably best. ${ }^{26}$

\section{The pathoanatomy of instability of the shoulder}

The shallow glenohumeral joint sacrifices its stability in order to allow an extensive range of movement. The joint restraints are normally divided into static stabilisers such as the glenoid fossa, the labrum, the joint capsule and the glenohumeral ligament, and dynamic stabilisers including the rotator cuff, the long head of biceps and the stabilisers of the scapula. There is significant interaction between these restraints and their relative importance alters with changing positions of the arm. Knowledge of the anatomy and biomechanics of the shoulder has improved considerably in recent years with the increasing availability of MRI, more detailed biomechanical laboratory studies and the increasing use of arthroscopy. Studies of both normal and abnormal shoulders have allowed an improved appreciation of the complex factors contributing to glenohumeral instability. An overview of the pathoanatomy is given here, but a more extensive review of this complex area has been made by Levine and Flatow. ${ }^{27}$

The Bankart lesion, with avulsion of the anterior capsulolabral complex inferior to the equator of the glenoid, has traditionally been regarded as the essential lesion of anterior traumatic dislocation of the shoulder. Although this lesion is almost invariably present in patients with traumatic instability, ${ }^{28,29}$ it is now thought that it does not produce instability in isolation. ${ }^{30}$ As the arm is brought into the 'at-risk' position of greater abduction, the resistance to external rotation is provided by the anteroinferior structures, especially the inferior glenohumeral ligaments, which act like a hammock to retain the humeral head in place. ${ }^{31}$ Plastic deformation of these structures occurs during the initial dislocation before avulsion of the labrum and becomes progressively more severe with subsequent episodes of instability. ${ }^{32-34}$ Repair of the Bankart lesion alone is consequently thought to be insufficient to stabilise the joint and re-tensioning of the anteroinferior capsuloligamentous complex is now considered to be an important adjunctive procedure in patients with repeated episodes of instability. ${ }^{35}$

There is renewed interest in the importance of the morphology of the anteroinferior part of the bony glenoid rim in glenohumeral instability. Subtraction three-dimensional CT has been used to image the glenoids of normal volunteers and patients with glenohumeral instability. Half of the latter group had an avulsion of the osseous glenoid rim, a bony Bankart lesion, and a further $40 \%$ had evidence of erosion or compression of the rim with loss of the normal 'pear-shaped' configuration of the glenoid. A cadaver study $^{36}$ has shown that an osseous defect in which the width is at least $20 \%$ of the length of the glenoid can cause instability of the shoulder. Clinically, impression lesions and avulsion fractures have been associated with recurrent instability ${ }^{16,37}$ and failure of arthroscopic surgical stabilisa- 
tion. ${ }^{38}$ Patients with large anteroinferior osseous defects of the glenoid, the 'inverted pear' sign, may therefore be better served by an open bony procedure which directly addresses the defect in the glenoid, rather than a conventional softtissue stabilisation. ${ }^{38}$

An impression fracture of the humeral head (the HillSachs lesion) is present in most patients with anterior instability. It is usually relatively small and does not contribute to instability. However, when the defect is larger and involves more than $30 \%$ of the humeral articular surface, it may 'engage' with the anterior glenoid during external rotation of the shoulder causing re-dislocation. ${ }^{39}$

Other lesions associated with instability have only been recognised since the development of arthroscopy of the shoulder. These include humeral avulsion of the glenohumeral ligaments (HAGL lesion), which are typically recognised after first-time dislocations and probably represent a variant from the normal pattern of anterior capsular stretching or rupture, ${ }^{40}$ anterior labroligamentous periosteal sleeve avulsions (ALPSA lesion) in which the detached sleeve heals medially to the scapular neck, allowing excessive humeral translation, ${ }^{41}$ superior labral anterior and posterior detachment (SLAP lesion), which may occur in continuity with the inferior labral avulsion, ${ }^{42,43}$ and defects of the rotator interval. ${ }^{44,45}$ The exact importance of many of these lesions in the pathogenesis of shoulder instability is not fully understood at present.

\section{Treatment of the initial dislocation}

Clinical assessment and primary treatment. A detailed history and examination are important in the assessment of any patient presenting with a primary dislocation in the Emergency Department, with careful documentation of any neurovascular deficit. Although palsy of the axillary nerve is the most common nerve injury, many types of brachial plexus lesion and isolated nerve palsies have been described. Anteroposterior and modified axial radiographs ${ }^{46}$ are important in confirming the dislocation and the presence of any associated fractures before relocation. Reduction using the Hippocratic method with sedation and adequate muscle relaxation remains the most widely practised method, although many other techniques have been successfully used. ${ }^{47}$ After-manipulation radiographs are mandatory to confirm a congruent relocation and to reassess the position of any associated fractures.

Although some studies have suggested that prolonged immobilisation of the shoulder, ${ }^{48}$ restriction of activity or an intensive programme of rehabilitation ${ }^{6,49}$ may reduce the risk of recurrent instability, these measures have not been consistently effective., ${ }^{4,50}$ Restriction of shoulder movement in a sling for a period of two to three weeks followed by a graduated rehabilitation programme is the accepted management of a primary dislocation in the young patient.

During the period of immobilisation, the shoulder is normally held in the so-called 'safe' position of internal rota- tion, neutral flexion/abduction with the elbow flexed to $90^{\circ}$. Recent evidence has shown that immobilisation with the arm held in external rotation may reduce the risk of subsequent instability by approximating the Bankart lesion to the neck of the glenoid giving a greater likelihood of its anatomical healing. ${ }^{51}$ Early comparison of the rates of secondary instability after immobilisation in either the 'safe' position or in external rotation have yielded promising results in favour of the latter. ${ }^{52}$ Patient compliance may be an issue with this form of treatment, and independent validation of the initial results is awaited.

Primary surgical management. In theory, performing an early primary stabilisation after a first-time traumatic dislocation in patients at high risk of recurrent instability is a logical procedure since the tear of the glenoid labrum is fresh, and plastic deformation of the capsuloligamentous complex may be minimal. An open stabilisation procedure is unacceptable in most initial dislocations and has rarely been used. ${ }^{53-55}$ It was not until the development of arthroscopic techniques in the shoulder that the prophylactic surgical treatment became an option. Arthroscopic stabilisation has a higher degree of acceptability to the patient as a primary treatment, and, if delivered within two weeks of the injury, does not prolong the subsequent period of immobilisation or rehabilitation. However, the provision of an acute arthroscopic shoulder service is expensive. In addition to the training and specialist expertise, a modern arthroscopic 'stack' system with television monitor, a fluid-delivery pump system, arthroscopic electrocautery, instrumentation and implants are all required. The operation is often technically challenging, is not free from complications and, because of our inability to predict with certainty those who will develop instability, may be unnecessary in some patients. Proof of an economic and clinical benefit from the use of prophylactic surgery will be required for the technique to become more widely adopted as well as a cultural change towards the early referral of primary dislocators for this form of specialist surgery.

It has been suggested that early arthroscopic lavage may in itself be sufficient to reduce the risk of re-dislocation ${ }^{56,57}$ by clearing the acute haemarthrosis and joint effusion, ${ }^{58}$ thereby encouraging anatomical healing of the detached labrum. However, repair of the Bankart lesion to the decorticated rim of the glenoid is the usual primary arthroscopic procedure. Repair was initially attempted using metal staples but these have been largely superceded by either custom-designed tacks or suture anchors to achieve direct repair of the labrum, or anteroposterior pull-through transglenoid sutures with knots tied either over the infraspinatus fascia ${ }^{59}$ or to the posterior glenoid (Fig. 1). ${ }^{60}$ Many of the tacks and suture anchors are engineered from bioabsorbable materials because of concerns regarding the long-term effects of the use of metal implants in young individuals. The technology is still evolving, most notably with 'knotless' suture anchors and suture 'welding' systems, and it 


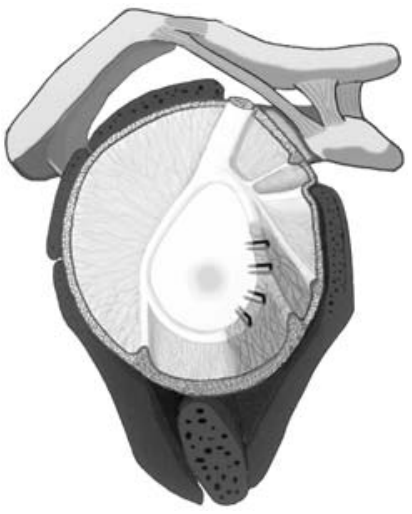

Fig. 1a

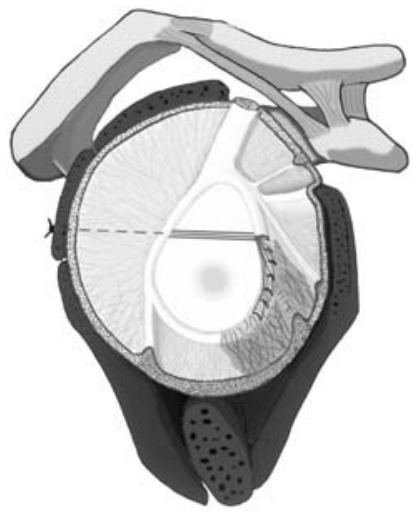

Fig. 1b

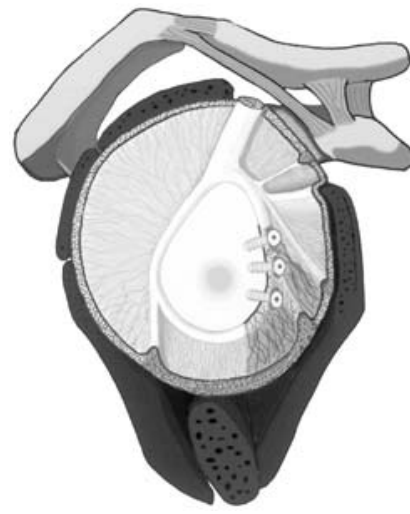

Fig. 1c

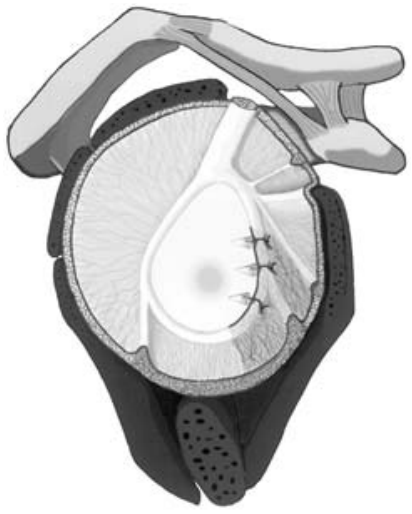

Fig. 1d

Diagrams of arthroscopic techniques for the treatment of anterior shoulder instability showing a) staple capsulorraphy, b) transglenoid suture repair, c) bioabsorbable tacks and d) suture anchors.

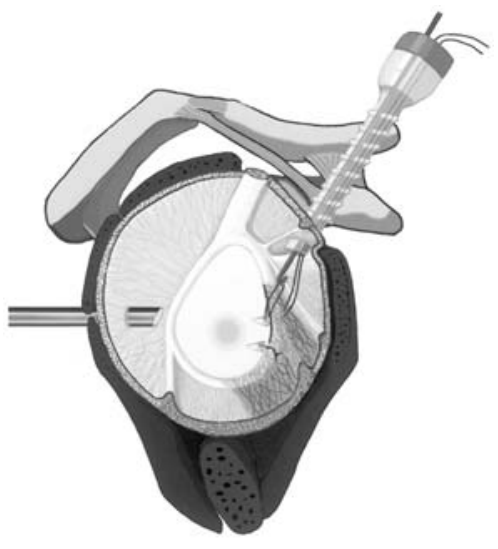

Fig. 2a

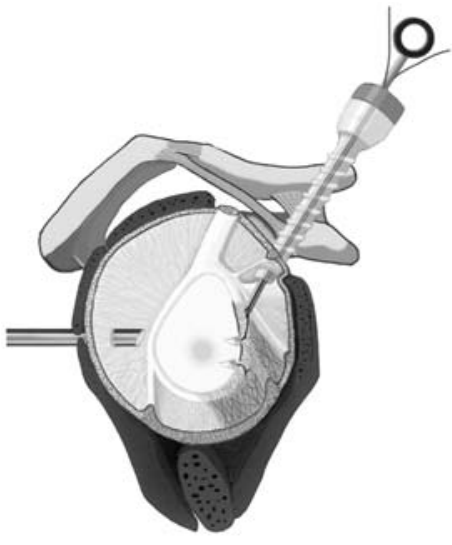

Fig. $2 b$

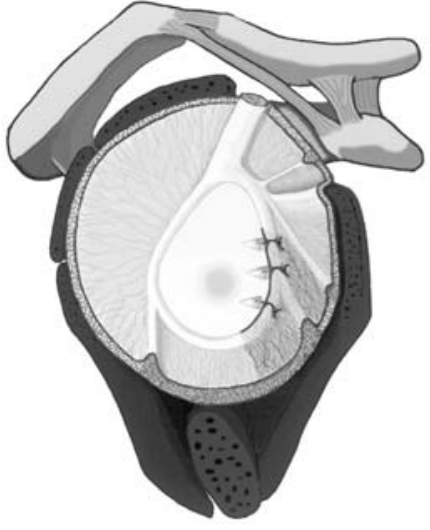

Fig. 2c

Diagrams of an acute arthroscopic repair using suture anchors: a) suture anchors have been placed into the anterior glenoid rim. The attached sutures penetrate the labrum and a crochet hook is used to pass one strand of the attached suture back through the anterior 'working' cannula to 'capture' the labrum; b) a knot pusher is used to tie an arthroscopic knot over the labrum; c) the completed repair.

seems certain that the procedure will become technically easier to perform in the future.

Arthroscopic stabilisation is often more technically challenging in the acute phase than when performed for recurrent instability (Fig. 2). Visualisation may be difficult because of the haemarthrosis, synovitis or active bleeding, and the shoulder may be relatively 'tight' and difficult to visualise. Rupture of the capsule may lead to excessive extravasation of arthroscopic fluid, the tissues may be friable and difficult to repair and the capsulolabral detachment may be more substantial (Fig. 3), with either global detachment extending into the superior and posterior labrum ${ }^{43}$ or an associated fracture of the glenoid rim. ${ }^{37}$

A number of relative contraindications to arthroscopic repair have been identified. These include 'red-out' at arthroscopy, usually due to active intra-articular bleeding preventing adequate visualisation and the presence of either a fracture of the greater tuberosity, a bony avulsion of the anterior glenoid rim or an HAGL lesion. Increasingly, these problems are being overcome by refinements to arthroscopic techniques. ${ }^{37,61-63}$

The results of primary surgical treatment. There are a number of concerns regarding previous studies which have examined the use of primary arthroscopic techniques. Many have been small case series without a control group. There are few comparative studies and even fewer prospective, randomised controlled trials which have compared arthroscopic stabilisation with standard non-operative treatment. Previous studies have varied widely in their criteria for inclusion and exclusion, the surgical techniques used, outcome measures, and length of follow-up. The chief outcome measure used has been the incidence of recurrent 


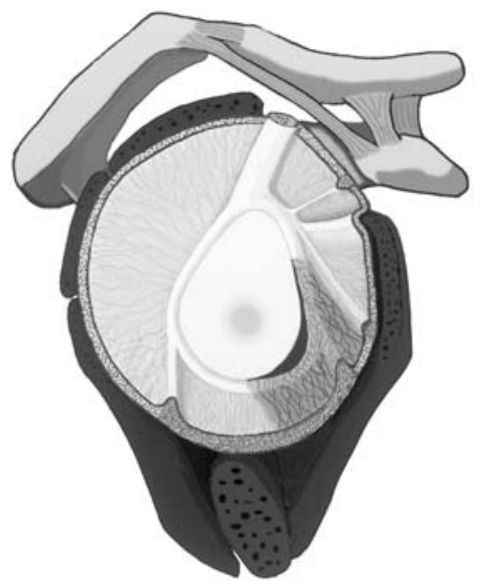

Fig. 3a

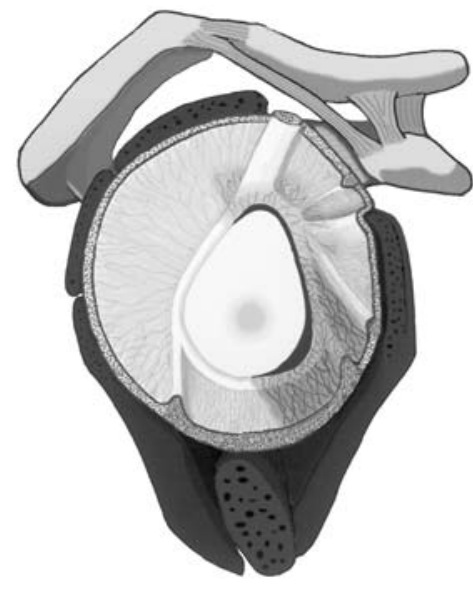

Fig. 3b

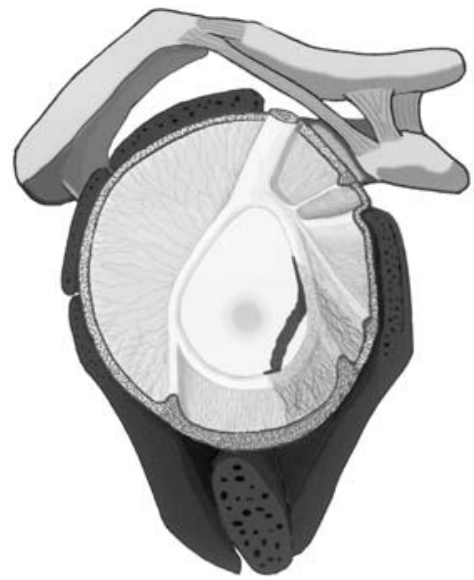

Fig. 3c

Diagrams of the variants of the Bankart lesion seen acutely showing a) an isolated Bankart lesion, b) a Bankart lesion in continuity with a SLAP lesion and c) a bony avulsion of the anterior glenoid rim ('bony Bankart lesion').

Table I. Rates of recurrence after the surgical treatment of patients with primary anteror dislocations and those with recurrent instability. This is a summary of the previous English Language articles, or articles with an English language abstract, which have reported on the effects of treatment for traumatic anterior shoulder instability in terms of recurrence post-operatively. In order to find all relevant articles, MEDLINE, EMBASE, PubMed and the Cochrane Central Register of Controlled Trials were searched. In addition, abstracts from past annual meetings of the American Academy of Orthopaedic Surgeons and the Arthroscopy Association of North America were searched. Review articles and the reference lists of articles were also checked. For the open stabilisation techniques, only studies published in the last ten years were included. Studies predominantly evaluating participants with posterior instability or multidirectional instability were excluded, as were those evaluating participants undergoing revision surgery. In addition, we specified a minimum mean follow-up period of 12 months. Post-surgical recurrence has been inconsistently defined, with studies variously including patients suffering re-dislocation, re-subluxation, post-surgical pain and those exhibiting positive apprehension signs. For the purpose of this review we defined post-surgical recurrence to be either redislocation or resubluxation

\begin{tabular}{|c|c|c|c|c|c|c|}
\hline \multirow{2}{*}{$\frac{\text { Clinical setting }}{\text { First-time dislocation }}$} & \multirow{2}{*}{$\begin{array}{l}\text { Type of study } \\
\text { Case-series (with no } \\
\text { control gp) }\end{array}$} & \multirow{2}{*}{$\begin{array}{l}\text { Technique } \\
\text { Arthroscopic Bankart repair }\end{array}$} & $\begin{array}{l}\text { Total number of } \\
\text { patients in } \\
\text { series }\end{array}$ & $\begin{array}{l}\text { Total } \\
\text { number of } \\
\text { recurrences }\end{array}$ & \multicolumn{2}{|c|}{$\begin{array}{l}\text { Percentage recurrence } \\
\text { rate (with } 95 \% \mathrm{CI} \text { ) }\end{array}$} \\
\hline & & & 170 & 13 & 7.6 & (4.5 to 12.6 ) \\
\hline & Clinical trials & $\begin{array}{l}\text { Non-operative } \\
\text { Arthroscopic Bankart repair }\end{array}$ & $\begin{array}{l}148 \\
177\end{array}$ & $\begin{array}{r}118 \\
23\end{array}$ & $\begin{array}{l}79.7 \\
13.0\end{array}$ & $\begin{array}{l}(72.5 \text { to } 85.4) \\
\text { (8.8 to } 18.7)\end{array}$ \\
\hline \multirow[t]{5}{*}{ Recurrent instability } & $\begin{array}{l}\text { Case-series (with no } \\
\text { control gp) }\end{array}$ & $\begin{array}{l}\text { Arthroscopic staple repair } \\
\text { Arthroscopic transglenoid suture repair } \\
\text { Arthroscopic bioabsorbable tack repair } \\
\text { Arthroscopic suture anchor repair } \\
\text { Arthroscopic repairs with adjunctive procedures }\end{array}$ & $\begin{array}{r}842 \\
1755 \\
616 \\
692 \\
141\end{array}$ & $\begin{array}{r}151 \\
302 \\
84 \\
68 \\
9\end{array}$ & $\begin{array}{r}17.9 \\
17.2 \\
13.6 \\
9.8 \\
6.4\end{array}$ & $\begin{array}{l}(15.5 \text { to } 20.7) \\
(15.5 \text { to } 19.0) \\
(11.2 \text { to } 16.6) \\
\text { (7.8 to } 12.3) \\
\text { (3.4 to } 11.7)\end{array}$ \\
\hline & & All arthroscopic repairs & 4046 & 614 & 15.2 & (14.1 to 16.3 ) \\
\hline & & $\begin{array}{l}\text { Open Bankart repair } \\
\text { Other open stabilisation techniques }\end{array}$ & $\begin{array}{l}1451 \\
1295\end{array}$ & $\begin{array}{r}91 \\
151\end{array}$ & $\begin{array}{r}6.3 \\
11.7\end{array}$ & $\begin{array}{l}(5.1 \text { to } 7.7) \\
(9.6 \text { to } 12.8)\end{array}$ \\
\hline & & All open stabilisation procedures & 2746 & 242 & 8.8 & (7.8 to 9.9$)$ \\
\hline & Clinical trials & $\begin{array}{l}\text { Open Bankart repair } \\
\text { Arthroscopic Bankart repair }\end{array}$ & $\begin{array}{l}529 \\
506\end{array}$ & $\begin{array}{l}37 \\
94\end{array}$ & $\begin{array}{r}7.0 \\
18.6\end{array}$ & $\begin{array}{l}(5.1 \text { to } 9.5) \\
(15.4 \text { to } 22.2)\end{array}$ \\
\hline
\end{tabular}

instability after operation. The use of this as the benchmark of the success of the technique is somewhat simplistic, although these patients usually score poorly in terms of the other outcome measures which have commonly been used, including levels of pain, subjective instability, functional outcome and the range of the shoulder movement. A summary of the overall rates of recurrence after operation reported in the literature is shown in Table I.

Case series of the use of primary arthroscopic Bankart repair without control groups report low rates of recurrence, comparable with those achieved after open repair to treat recurrent instability (Table I). However, in the studies which have compared primary arthroscopic stabilisation with non-operative treatment, the incidence of recurrent instability after surgery has been much higher (Table I). Despite this, the evidence of these studies still points to a more than fivefold reduction in the risk of recurrent instability after arthroscopic stabilisation, when compared with non-operative treatment (Fig. 4). It is notable that the rates of recurrence after operation which are reported after primary arthroscopic repair are similar to those after arthroscopic stabilisation to treat recurrent instability (Table I). 


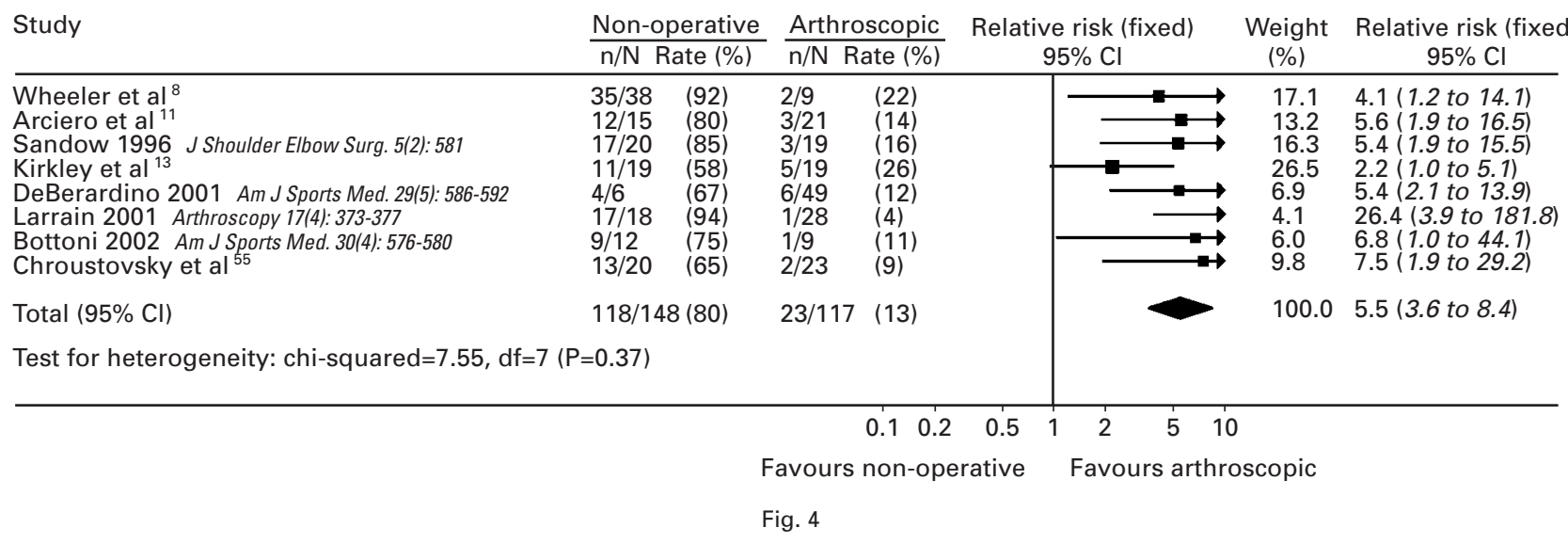

Summary of the previous English language articles, or articles with an English language abstract, which have compared the effects of arthroscopic and traditional non-operative treatment for primary anterior dislocation of the shoulder in terms of recurrence postoperatively. In order to find all relevant articles, MEDLINE, EMBASE, PubMed and the Cochrane Central Register of Controlled Trials were searched. In addition, abstracts from past annual meetings of the American Academy of Orthopaedic Surgeons and the Arthroscopy Association of North America were searched. Review articles and the reference lists of articles were also checked. Due to the lack of randomised and quasi-randomised trials which have been conducted in this area, it was decided to include all types of clinical trial. Studies predominantly evaluating participants with posterior instability or multidirectional instability were excluded. In addition, we specified a minimum mean follow-up period of 12 months. Postsurgical recurrence has been inconsistently defined, with studies variously including patients suffering re-dislocation, re-subluxation, post-surgical pain and those showing positive apprehension signs. For the purposes of the analysis we defined post-surgical recurrence to be either redislocation or resubluxation. The figures to the right of the reference show the number of patients with instability postoperatively ( $\mathrm{n}$ ) as a proportion of the total population ( $\mathrm{N}$ ). The chi-squared test showed evidence of some statistical heterogeneity of the studies, demonstrated by a chi-square statistic greater than its degree of freedom (df). However, this is not statistically significant $(p=0.37)$. The results suggest that the risk of recurrent post-operative instability is 5.5 times greater after non-operative treatment compared with primary arthroscopic stabilisation. (relative risk=5.5, 95\% confidence levels (Cl) 3.6 to 8.4 ).

Primary arthroscopic stabilisation has shown no consistent benefit over non-operative treatment in terms of other functional outcomes including levels of shoulder pain, functional scores or restriction of movement.

\section{Treatment of the patient with recurrent instability} Clinical assessment. The clinical assessment of the patient presenting with symptoms of instability is primarily directed towards identifying those individuals with predominantly traumatic instability, who would benefit from surgical treatment. These patients need to be distinguished from those with predominantly atraumatic or voluntary instability, who are best treated non-operatively in the first instance, and from those with more subtle forms of instability who require further investigation before a decision can be made about treatment.

Details about the onset, duration and frequency of the symptoms should be sought in the history. Physical examination should include screening for evidence of generalised ligamentous laxity ${ }^{64,65}$ and performing provocative tests to define the direction and extent of instability.

Plain radiography is useful in delineating any associated bony abnormality, including defects of the rim of the glenoid and head of the humerus. Further specialist radiological investigation, examination under anaesthesia or diagnostic arthroscopy may be used for patients in whom the precise diagnosis is in doubt. MRI of the shoulder is superior to CT in the assessment of instability of the shoulder because of the better soft-tissue definition provided.
Surgical treatment for recurrent instability. A programme of physiotherapy rehabilitation alone will not usually restore sufficient stability to allow an athlete with traumatic instability to return to physical contact sports. ${ }^{66}$ The mainstay of treatment in these individuals is surgical stabilisation. This may not apply to more sedentary individuals, to those with occasional symptoms of instability or to older patients. In these individuals, an initial trial of non-operative treatment including strengthening of the rotator cuff may be more appropriate.

Open surgical stabilisation. Irrespective of the exact technique used, there has been a general trend towards attempting to identify and treat the lesion responsible for instability by using anatomical repair and re-tensioning of soft tissues, rather than achieving stability through scarring or restriction of movement.

During the last 20 years, the most commonly used open technique in the treatment of recurrent anterior instability has been repair of the Bankart lesion, usually combined with an adjuvant capsular shift. The deltopectoral approach is used almost exclusively, but a more cosmetic and minimally-invasive vertical skin incision extending in the skin crease from the anterior axillary fold is now recommended. Most studies advocate dissecting the subscapularis tendon as a separate layer before performing a capsulotomy. Several varieties of T-shaped, vertical or horizontal incisions have been described to perform the capsulotomy. Many variations of the Bankart repair have been used, but most surgeons now tend to favour suture anchors to secure the glenoid labrum to the decorticated anterior rim of the 


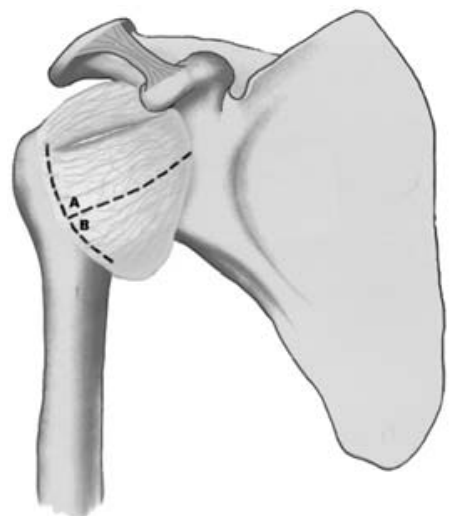

Fig. 5a

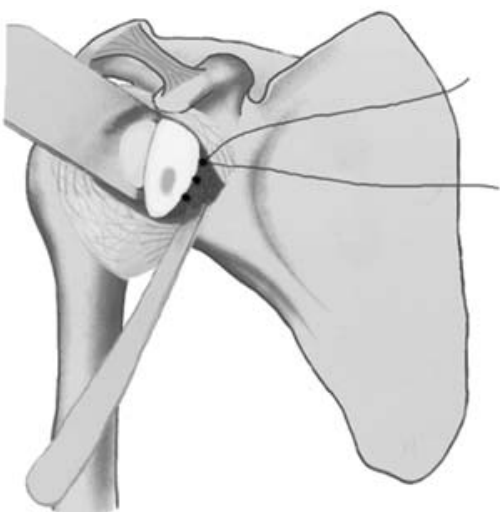

Fig. $5 b$

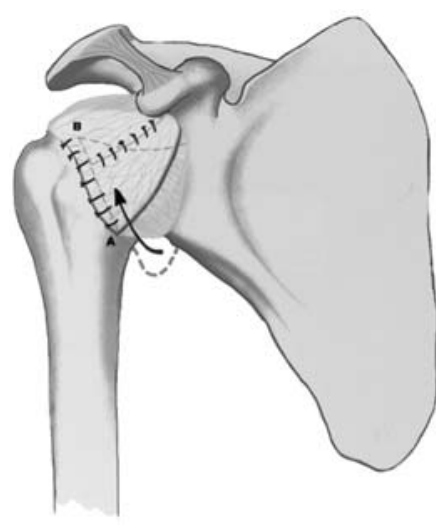

Fig. $5 \mathrm{c}$

Diagrams of an open Bankart repair with capsular shift. (a) The t-shaped capsular incision (note the rotator interval defect and large inferior capsular pouch). (b) A capsulotomy has been performed and drill holes have been inserted into the decorticated anterior glenoid rim for placement of suture anchors to repair the labral avulsion (Bankart repair). (c) On completion of the Bankart repair, a capsular shift procedure is performed to re-tension the lax anterior capsuloligamentous structures and obliterate the inferior capsular recess. The rotator interval has also been closed.

glenoid (Fig. 5). A plication is performed to re-tension the anteroinferior capsuloligamentous complex. Care must be taken not to overtighten the repair in order to avoid the restriction of external rotation post-operatively.

Many other open techniques are still widely used, with a high degree of success. ${ }^{67,68}$ The Bristow-Helfet procedure of coracoid osteotomy and fixation to the anteroinferior rim of the glenoid, although technically demanding, remains popular. ${ }^{69,70}$ Many consider that this procedure, or the use of a structural bone graft to reconstitute the rim of the glenoid, is preferable for patients with a large defect in the rim. This applies particularly to epileptics with recurrent traumatic instability who often have a large anteroinferior deficiency. ${ }^{71,72}$ In patients with large, engaging HillSachs defects, allograft bone grafting or rotational osteotomy may also be considered.

Arthroscopic stabilisation. The arthroscopic techniques used to repair the Bankart lesion in the treatment of recurrent traumatic instability are similar to those advocated for primary stabilisation. However, in patients with recurrent instability, the anteroinferior capsule becomes progressively more attenuated with each episode of subluxation or dislocation. Tightening of the anterior capsule, achieved either by superior advancement of the labrum at the time of Bankart repair or as a separate adjunctive thermal or laser-assisted shrinkage of the anterior capsule, suture plication of the capsule or closure of the rotator interval are therefore increasingly advocated. Judging the extent to which this is required may be difficult and incurs the risk of producing excessive capsular tightness, limiting external rotation. Thermal or laser-assisted capsular shrinkage may initially reduce laxity but it may engender an increased susceptibility to creep failure at low physiological loads. ${ }^{73,74}$
The results of stabilisation procedures for recurrent traumatic instability. The most widely reported results using open techniques are those for the Bankart repair and are slightly better than those for other open procedures (Table I). However, combination of all the reported series using open techniques during the last ten years still reveals a rate of recurrence after operation of less than $10 \%$. Notable success has been achieved recently using open Bankart repair to treat high-demand, collision sportsmen with unstable shoulders, with most returning successfully to high-level competition. ${ }^{75,76}$

If arthroscopic stabilisation procedures are to replace open procedures, the rates of recurrence after operation need to be similar. The overall average rate of recurrence after arthroscopic stabilisation in case series without control groups is $15.2 \%$, compared with $6.3 \%$ for open Bankart repair and $8.8 \%$ for all open procedures (Table I). However, the rates of recurrence vary considerably between the four categories of arthroscopic techniques and there is often considerable heterogenicity in the results of studies reporting the same method. Although the rates of recurrence are greater in studies with longer follow-up, there appears to have been a gradual improvement with the use of the more modern arthroscopic techniques and implants, particularly suture anchors. The few available studies suggest that the results of arthroscopic Bankart repair with a separate adjuvant capsular tightening, either by closure of the rotator interval, capsular shrinkage or plication, may be better than a Bankart repair alone (Table I).

Most of the comparative studies have shown rates of recurrent instability to be higher after arthroscopic stabilisation than after open repair, and a meta-analysis of these series shows the risk of recurrence to be statistically significant, and more than twice that of an open procedure (Fig. 6). 


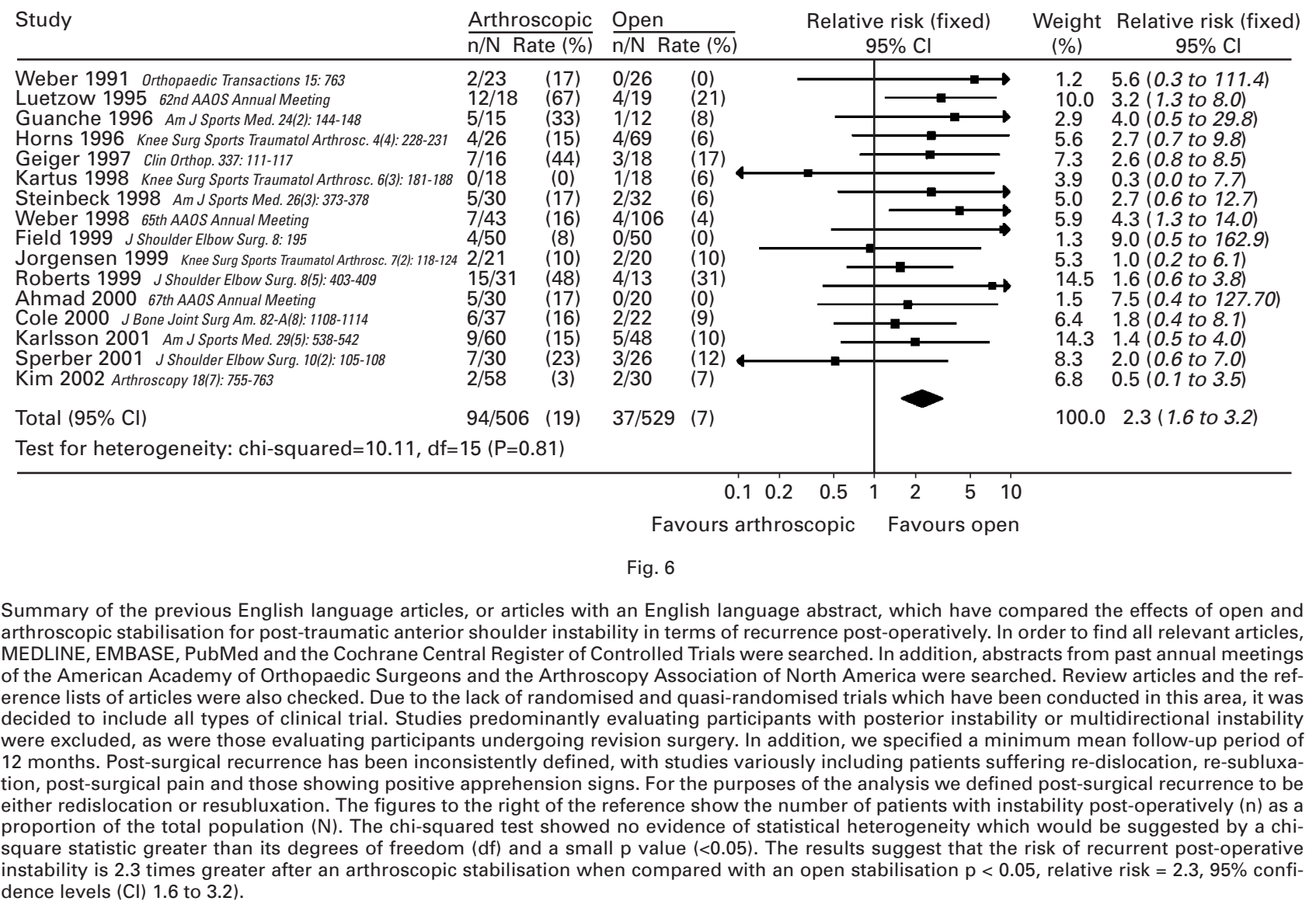

As with primary stabilisation, no consistent benefits of the arthroscopic procedure over an open stabilisation have been demonstrated in terms of long-term residual shoulder pain, functional scores or restriction of movement. However, many studies report a shorter hospital stay, decreased post-operative pain and analgesic requirements, together with an improved cosmetic result when using the arthroscopic method.

\section{Factors associated with recurrent instability after surgical repair}

The factors associated with recurrent instability after surgical stabilisation may be classified into those caused by inappropriate patient selection and those attributable to surgical error. ${ }^{77,78}$ However, athletes playing contact sports may often re-injure themselves with sufficient force to redislocate their shoulder irrespective of the quality of their previous repair. ${ }^{79,80}$

Failure because of inappropriate patient selection usually results from inadequate pre-operative assessment. The most common cause of this is a failure to recognise a multidirectional or voluntary element in a patient thought to have anterior instability. The most common reason for surgical error is inadequate treatment of all the constituent components of the instability at the time of surgery. Abnor- malities commonly encountered at re-exploration after failed arthroscopic or open repair include an unhealed Bankart lesion, ${ }^{77,81}$ humeral avulsion of the glenohumeral ligaments, ${ }^{82,83}$ extensive glenoid erosion or deficiency from a bony Bankart lesion, ${ }^{38,84,85}$ excessive capsular laxity, ${ }^{81,86}$ a defect of the rotator interval, ${ }^{87}$ an engaging Hill-Sachs lesion $^{38,39}$ and reduced retroversion of the head of the humerus or excessive retroversion of the glenoid cavity. ${ }^{88}$

Several additional factors have been associated with recurrent instability after arthroscopic stabilisation including a younger age at surgery, ${ }^{59,89}$ non-compliance with post-operative immobilisation, ${ }^{84,90}$ early return to contact sport, ${ }^{86,91}$ absence or deficiency of the capsulolabral complex and poor inferior glenohumeral ligaments, ${ }^{86,92}$ and multiple episodes of instability before stabilisation. ${ }^{80,93}$ The wide variation in the rates of recurrence after arthroscopic stabilisation may also be a reflection of the inherent technical difficulty in performing these procedures. Many authors report a steep learning curve, with patients included later in their series having a lower rate of recurrence than those treated earlier.

Although revision arthroscopic stabilisation has been described, ${ }^{86,94}$ most failed open and arthroscopic stabilisations caused by technical failure are treated by a further open procedure. ${ }^{95}$ High-quality MRI should be performed 
before re-exploration to delineate abnormalities of bone and soft tissue clearly and to help to plan surgery. An examination under anaesthesia and diagnostic arthroscopy will often provide extra information before re-exploration. Specific guidelines for the treatment of postsurgical recurrence have previously been described ${ }^{68}$ but a flexible approach is important, as every case is idiosyncratic.

\section{Other complications of surgical stabilisation}

In addition to the general complications of surgery and recurrent instability, a number of other problems have been described after operative stabilisation. Following open repair, pain, loss of movement, infection, failure of the implant, neurovascular injury and late degenerative joint disease are the more commonly encountered complications. ${ }^{68,96}$ After arthroscopic stabilisation, neurovascular injury, ${ }^{97-99}$ adhesive capsulitis ${ }^{100}$ and synovial fistula ${ }^{101}$ have all been encountered. Other complications are specific to the particular technique used to repair the Bankart lesion and include loosening, breakage and impingement after the use of staples; $;^{102,103}$ injury to the suprascapular nerve and pain from the posterior knot after transglenoid repair ${ }^{101,104}$ foreign-body reaction, rapid decay leading to intra-articular dissociation of the head after repair with bioabsorbable tacks; ${ }^{105,106}$ and pull-out after repair with suture anchors. ${ }^{80}$ These complications are comparatively rare, but often require surgical re-exploration and are associated with a poor functional outcome or recurrent instability.

\section{Conclusions and the future}

The last 20 years have seen many advances in our understanding and treatment of post-traumatic instability of the shoulder. Innovative treatments designed either to prevent or to treat recurrent instability vary from those which are relatively simple, inexpensive and require little expertise, such as, immobilisation of the shoulder in external rotation and arthroscopic washout of the shoulder alone, to the more technically-demanding and financially-burdensome procedures for arthroscopic stabilisation.

The challenge for the future rests in defining the most appropriate timing and type of intervention from the range of new and traditional options available. The current literature contains many small case series and comparative studies, but larger collaborative, randomised controlled trials are required to compare the efficacy of the newer techniques with standard treatments. The methods chosen will vary from patient to patient dependant on their age, their degree of activity and their expectations from treatment. Ultimately, a greater degree of patient empowerment in the decision-making process may be the way forward. ${ }^{107}$

Until good evidence is produced to the contrary the standard method of treatment of an initial traumatic dislocation is non-operative and open repair is used to treat most patients with recurrent traumatic instability. Currently, there is some evidence that early arthroscopic stabi- lisation can substantially reduce the risk of recurrent instability after a primary dislocation. However, the risk of recurrence after an immediate arthroscopic stabilisation in these studies does not appear to be substantially better than that for an arthroscopic procedure to treat patients with recurrent instability. With the inherent degree of 'overtreatment' involved in offering an immediate arthroscopic stabilisation to all high-risk patients, an effective compromise may be to offer this procedure at a much earlier stage to those individuals who develop instability after a primary dislocation, before they have developed the degree of capsuloligamentous plastic deformation likely to compromise an arthroscopic repair.

Many patients still present late, with multiple episodes of instability, and either attenuated capsuloligamentous restraints or a deficiency of the anteroinferior glenoid rim. The results of arthroscopic stabilisation in these patients are improving with the use of newer implants and adjuvant treatment to address the problem of anterior capsular laxity. However, currently there is little evidence to support the routine widespread use of this technique over a standard open stabilisation and at the moment most patients with recurrent traumatic instability are better served by an open Bankart repair with re-tensioning of the capsule.

\section{References}

1. Gumina S, Postacchini F. Anterior dislocation of the shoulder in elderly patients. J Bone Joint Surg [Br] 1997;79-B:540-3.

2. Rowe CR. Prognosis in dislocations of the shoulder. J Bone Joint Surg [Am] 1956 38-A:957-77.

3. Hovelius $\mathbf{L}$, Lind $\mathbf{B}$, Thorling J. Primary dislocation of the shoulder: factors affecting the two-year prognosis. Clin Orthop 1983;176:181-5.

4. Hovelius L, Augustini BG, Fredin H, et al. Primary anterior dislocation of the shoulder in young patients: a ten-year prospective study. J Bone Joint Surg [Am] 1996;78-A:1677-84.

5. Simonet WT, Cofield RH. Prognosis in anterior shoulder dislocation. Am J Sports Med 1984;12:19-24.

6. Yoneda B, Welsh RP, MacIntosh DL. Conservative treatment of shoulder dislocation in young males. J Bone Joint Surg [Br] 1982;64-B:254-5.

7. Henry JH, Genung JA. Natural history of glenohumeral dislocation-revisited. Am J Sports Med 1982;10:135-7.

8. Wheeler JH, Ryan JB, Arciero RA, Molinari RN. Arthroscopic versus nonoperative treatment of acute shoulder dislocation in young athletes. Arthroscopy 1989;5: 213-17.

9. Marans HJ, Angel KR, Schemitsch EH, Wedge JH. The fate of traumatic anterior dislocation of the shoulder in children. J Bone Joint Surg [Am] 1992;74-A:1242-4.

10. Postacchini F, Gumina S, Cinotti G. Anterior shoulder dislocation in adolescents J Shoulder Elbow Surg 2000;9:470-4.

11. Arciero RA, Wheeler JH, Ryan JB, McBride JT. Arthroscopic Bankart repair versus nonoperative treatment for acute, initial anterior shoulder dislocations. Am J Sports Med 1994;22:589-94.

12. Hovelius L. Incidence of shoulder dislocation in Sweden. Clin Orthop 1982;66: 127-31.

13. Kirkley A, Griffin S, Richards C, Miniaci A, Mohtadi N. Prospective randomized clinical trial comparing the effectiveness of immediate arthroscopic stabilization versus immobilization and rehabilitation in first traumatic anterior dislocations of the shoulder. Arthroscopy 1999;15:507-14.

14. Kralinger FS, Golser K, Wischatta R, Wambacher M, Sperner G. Predicting recurrence after primary anterior shoulder dislocation. Am J Sports Med 2002;30:116-20

15. O'Driscoll SW, Evans DC. Contralateral shoulder instability following anterior repair: an epidemiological investigation. J Bone Joint Surg [Br] 1991;73-B:941-6.

16. Bigliani LU, Newton PM, Steinmann SP, Connor PM, Mcllveen SJ. Glenoid rim lesions associated with recurrent anterior dislocation of the shoulder. Am J Sports Med 1998;26:41-5. 
17. McLaughlin HL, Cavallaro WV. Primary anterior dislocation of the shoulder. Am J Surg 1950;80:615-21.

18. Harryman DT, Sidles JA, Harris SL, et al. Laxity of the normal glenohumeral joint: a quantitative in vivo assessment. J Shoulder Elbow Surg 1992;1:66-76.

19. Thomas SC, Matsen FA, 3rd. An approach to the repair of avulsion of the glenohumeral ligaments in the manageent of traumatic anterior glenohumeral instability. J Bone Joint Surg [Am]1989;71-A:506-13.

20. Dowdy PA, O'Driscoll SW. Shoulder instability: an analysis of family history. J Bone Joint Surg [Br] 1993;75-B:782-4.

21. Jobe FW, Kvitne RS, Giangarra CE. Shoulder pain in the overhand or throwing athlete: the relationship of anterior instability and rotator cuff impingement. Orthop Rev 1989;18:963-75

22. Kvitne RS, Jobe FW. The diagnosis and treatment of anterior instability in the throwing athlete. Clin Orthop 1993;291:107-23.

23. Kvitne RS, Jobe FW, Jobe CM. Shoulder instability in the overhand or throwing athlete. Clin Sports Med 1995;14:917-35.

24. Rowe CR, Pierce DS, Clark JG. Voluntary dislocation of the shoulder: a preliminary report on a clinical, electromyographic, and psychiatric study of twenty-six patients. $J$ Bone Joint Surg [Am] 1973;55-A:445-60.

25. Fuchs B, Jost B, Gerber C. Posterior-inferior capsular shift for the treatment of recurrent, voluntary posterior subluxation of the shoulder. J Bone Joint Surg [Am] 2000;82-A:16-25

26. Silliman JF, Hawkins RJ. Classification and physical diagnosis of instability of the shoulder. Clin Orthop 1993;291:7-19.

27. Levine WN, Flatow EL. The pathophysiology of shoulder instability. Am J Sports Med 2000;28:910-17

28. Baker CL, Uribe JW, Whitman C. Arthroscopic evaluation of acute initial anterior shoulder dislocation. Am J Sports Med 1990;18:25-8.

29. Taylor DC, Arciero RA. Pathologic changes associated with shoulder dislocations: arthroscopic and physical examination findings in first-time, traumatic anterior dislocations. Am J Sports Med 1997;25:306-11.

30. Speer KP, Deng X, Borrero S, et al. Biomechanical evaluation of a simulated Bankart lesion. J Bone Joint Surg [Am] 1994;76-A:1819-26.

31. Turkel SJ, Panio MW, Marshall JL, Girgis FG. Stabilizing mechanisms preventing anterior dislocation of the glenohumeral joint. J Bone Joint Surg [Am] 1981;63-A: 1208-17.

32. Bigliani LU, Pollock RG, Soslowsky LJ, et al. Tensile properties of the inferior glenohumeral ligament. J Orthop Res 1992;10:187-97.

33. Habermeyer $\mathbf{P}$, Gleyze $\mathbf{P}$, Rickert $\mathbf{M}$. Evolution of lesions of the labrum-ligament complex in posttraumatic anterior shoulder instability: a prospective study. J Shoulder Elbow Surg 1999;8:66-74.

34. Urayama M, Itoi E, Sashi R, Minagawa H, Sato K. Capsular elongation in shoulders with recurrent anterior dislocation: quantitative assessment with magnetic resonance arthrography. Am J Sports Med 2003;31:64-7.

35. Bigliani LU, Kurzweil PR, Schwartzbach CC, Wolfe IN, Flatow EL. Inferior capsular shift procedure for anterior-inferior shoulder instability in athletes. Am J Sports Med 1994;22:578-84

36. Itoi E, Lee SB, Berglund LJ, Berge LL, An KN. The effect of a glenoid defect on anteroinferior stability of the shoulder after Bankart repair: a cadaveric study. J Bone Joint Surg [Am]2000;82-A:35-46

37. Porcellini G, Campi F, Paladini P. Arthroscopic approach to acute bony Bankart lesion. Arthroscopy 2002;18:764-9.

38. Burkhart SS, De Beer JF. Traumatic glenohumeral bone defects and their relationship to failure of arthroscopic Bankart repairs: significance of the inverted-pear glenoid and the humeral engaging Hill-Sachs lesion. Arthroscopy 2000;16:677-94.

39. Rowe CR, Zarins B, Ciullo JV. Recurrent anterior dislocation of the shoulder after surgical repair: apparent causes of failure and treatment. J Bone Joint Surg [Am] 1984;66-A:159-68

40. Wolf EM, Cheng JC, Dickson K. Humeral avulsion of glenohumeral ligaments as a cause of anterior shoulder instability. Arthroscopy 1995;11:600-7.

41. Neviaser TJ. The anterior labroligamentous periosteal sleeve avulsion lesion: a cause of anterior instability of the shoulder. Arthroscopy 1993;9:17-21.

42. Snyder SJ, Karzel RP, Del Pizzo W, Ferkel RD, Friedman MJ. SLAP lesions of the shoulder. Arthroscopy 1990;6:274-9.

43. Warner JJ, Kann S, Marks P. Arthroscopic repair of combined Bankart and superior labral detachment anterior and posterior lesions: technique and preliminary results. Arthroscopy 1994;10:383-91

44. Harryman DT 2nd, Sidles JA, Harris SL, Matsen FA 3rd. The role of the rotator interval capsule in passive motion and stability of the shoulder. $J$ Bone Joint Surg [Am] 1992;74-A:53-66.

45. Field LD, Warren RF, O'Brien SJ, Altchek DW, Wickiewicz TL. Isolated closure of rotator intervals defects for shoulder instability. Am J Sports Med 1995;23: 557-63.
46. Wallace WA, Hallier M. Improving radiographs of the injured shoulder. Radiography 1983:49:229-33.

47. Riebel GD, McCabe JB. Anterior shoulder dislocation: a review of reduction techniques. Am J Emerg Med 1991;9:180-8.

48. Kiviluoto $\mathbf{0}$, Pasila M, Jaroma H, Sundholm A. Immobilization after primary dislocation of the shoulder. Acta Orthop Scand 1980;51:915-19.

49. Aronen JG, Regan K. Decreasing the incidence of recurrence of first time anterior shoulder dislocations with rehabilitation. Am J Sports Med 1984;12:283-91.

50. Hoelen MA, Burgers AM, Rozing PM. Prognosis of primary anterior shoulder dislocation in young adults. Arch Orthop Trauma Surg 1990;110:51-4.

51. Itoi E, Sashi R, Minagawa H, et al. Position of immobilization after dislocation of the glenohumeral joint: a study with use of magnetic resonance imaging. J Bone Joint Surg [Am]2001;83-A:661-7.

52. Itoi E, Hatakeyama Y, Kido T, et al. A new method of immobilisation after dislocation of the shoulder: a prospective randomised study: 2003: Procs American Academy of Orthopaedic Surgeons Meeting, New Orleans 2003.

53. Nicola T. Acute anterior dislocation of the shoulder. J Bone Joint Surg [Am] 1949; 31-A:153-9.

54. Ambacher T, Paar 0. Traumatic shoulder dislocation in young athletes-open or arthroscopic stabilization? Sportverletz Sportschaden 1999:13:68-73.

55. Chroustovsky J, Malusek P, Jircik M, Konecny R. Treatment of anterior glenohumeral instability: personal experience with an arthroscopic stabilization technique, its indications and results. Acta Chir Orthop Traumatol Cech 2003;70:164-9.

56. Wintzell G, Haglund-Akerlind Y, Ekulund A, et al. Arthroscopic lavage reduced the recurrence rate following primary anterior shoulder dislocation: a randomised multicentre study with 1-year follow-up. Knee Surg Sports Traumatol Arthrosc 1999; 7:192-6

57. Wintzell G, Haglund-Akerlind Y, Nowak J, Larsson S. Arthroscopic lavage compared with nonoperative treatment for traumatic primary anterior shoulder dislocation: a 2-year follow-up of a prospective randomized study. J Shoulder Elbow Surg 1999;8:399-402.

58. Wintzell G, Hovelius L, Wikblad L, Saebo M, Larsson S. Arthroscopic lavage speeds reduction in effusion in the glenohumeral joint after primary anterior shoulder dislocation: a controlled randomized ultrasound study. Knee Surg Sports Traumatol Arthrosc 2000;8:56-60.

59. Torchia ME, Caspari RB, Asselmeier MA, Beach WR, Gayari M. Arthroscopic transglenoid multiple suture repair: 2 to 8 year results in 150 shoulders. Arthroscopy 1997:13:609-19.

60. Yoneda M, Hayashida K, Izawa K, Shimada K, Shino K. A simple and secure anchoring system for Caspari's transglenoid multiple suture technique using a biodegradable poly-I-lactic acid button. Arthroscopy 1996;12:293-9.

61. Cameron SE. Arthroscopic reduction and internal fixation of an anterior glenoid fracture. Arthroscopy 1998;14:743-6.

62. Kim SH, Ha KI. Arthroscopic treatment of symptomatic shoulders with minimally displaced greater tuberosity fracture. Arthroscopy 2000;16:695-700.

63. Bonsell S, Buford DA Jr. Arthroscopic reduction and internal fixation of a greater tuberosity fracture of the shoulder: a case report. J Shoulder Elbow Surg 2003;12: $397-400$

64. McFarland EG, Torpey BM, Curl LA. Evaluation of shoulder laxity. Sports Med 1996;22:264-72

65. Cofield RH, Irving JF. Evaluation and classification of shoulder instability with special reference to examination under anaesthesia. Clin Orthop 1987;223:32-43.

66. Burkhead WZ Jr, Rockwood CA Jr. Treatment of instability of the shoulder with an exercise program. J Bone Joint Surg [Am] 1992;74-A:890-6.

67. Payne LZ, Altchek DA. The surgical treatment of anterior shoulder instability. Clin Sports Med 1995:14:863-83.

68. Gill TJ, Zarins B. Open repairs for the treatment of anterior shoulder instability. Am J Sports Med 2003;31:142-53.

69. Wredmark T, Tornkvist H, Johansson C, Brobert B. Long-term functional results of the modified Bristow procedure for recurrent dislocations of the shoulder. Am J Sports Med 1992;20:157-61.

70. Yamashita T, Okamura K, Hotta T, et al. Good clinical outcome of combined Bankart-Bristow procedure for recurrent shoulder instability: 126 patients followed for 2-6 years. Acta Orthop Scand 2002;73:553-7.

71. Hutchinson JW, Neumann L, Wallace WA. Bone buttress operation for recurrent anterior shoulder dislocation in epilepsy. J Bone Joint Surg [Br] 1995;77-B:928-32.

72. Buhler M, Gerber C. Shoulder instability related to epileptic seizures. J Shoulder Elbow Surg 2002;11:339-44.

73. Wallace AL, Hollinshead RM, Frank CB. Electrothermal shrinkage reduces laxity but alters creep behavior in a lapine ligament model. J Shoulder Elbow Surg 2001;10: $1-6$

74. Wallace AL, Hollinshead RM, Frank CB. Creep behavior of a rabbit model of ligament laxity after electrothermal shrinkage in vivo. Am J Sports Med 2002;30:98-102. 
75. Roberts SN, Taylor DE, Brown JN, Hayes MG, Saies A. Open and arthroscopic techniques for the treatment of traumatic anterior shoulder instability in Australian rules football players. J Shoulder Elbow Surg 1999;8:403-9.

76. Pagnani MJ, Dome DC. Surgical treatment of traumatic anterior shoulder instability in American football players. J Bone Joint Surg [Am] 2002;84-A:711-15.

77. Hawkins RH, Hawkins RJ. Failed anterior reconstruction for shoulder instability J Bone Joint Surg [Br] 1985;67-B:709-14.

78. Gill TJ, Warren RF, Rockwood CA Jr, et al. Complications of shoulder surgery. Instr Course Lect 1999;48:359-74.

79. Maracci M, Zaffagnini S, Petitto A, et al. Arthroscopic management of recurrent anterior dislocation of the shoulder: analysis of technical modifications on the Caspar procedure. Arthroscopy 1996;12:144-9.

80. Koss S, Richmond JC, Woodward JS Jr. Two- to five-year followup of arthroscopic Bankart reconstruction using a suture anchor technique. Am J Sports Med 1997:25:809-12

81. Mologne TS, McBride MT, Lapoint JM. Assessment of failed arthroscopic anterior labral repairs: findings at open surgery. Am J Sports Med 1997;25:813-17.

82. Bach BR, Warren RF, Fronek J. Disruption of the lateral capsule of the shoulder: cause of recurrent dislocation. J Bone Joint Surg [Br] 1988;70-B:274-6.

83. Bokor DJ, Conboy VB, OIson C. Anterior instability of the glenohumeral joint with humeral avulsion of the glenohumeral ligament: a review of 41 cases. J Bone Joint Surg $[\mathrm{Br}] 1999 ; 81-\mathrm{B}: 93-6$.

84. Grana WA, Buckley PD, Yates CK. Arthroscopic Bankart suture repair. Am J Sports Med 1993;21:348-53.

85. Walch G, Boileau P, Levigne C, et al. Arthroscopic stabilization for recurrent anterior shoulder dislocation: results of 59 cases. Arthroscopy 1995;11:173-9.

86. Warner JJ, Miller MD, Marks P, Fu FH. Arthroscopic Bankart repair with the Suretac device: Part I: clinical observations. Arthroscopy 1995;11:2-13.

87. Rowe CR, Zarins B. Recurrent transient subluxation of the shoulder. J Bone Joint Surg $[A m] 1981 ; 63-A: 863-71$

88. Zarins B, Rowe CR, Stone JW. Shoulder instability: management of failed reconstructions. Instr Course Lect 1989;38:217-30.

89. Savoie FH 3rd, Miller CD, Field LD. Arthroscopic reconstruction of traumatic anterior instability of the shoulder: the Caspari technique. Arthroscopy 1997;13:201-9.

90. Green MR, Christensen KP. Arthroscopic Bankart procedure: two- to five-year followup with clinical correlation to severity of glenoid labral lesion. Am J Sports Med 1995;23:276-81

91. Goldberg BJ, Nirschl RP, McConnell JP, Pettrone FA. Arthroscopic transglenoid suture capsulolabral repairs: preliminary results. Am J Sports Med 1993;21:656-64.
92. Manta JP, Organ S, Nirschle RP, Pettrone FA. Arthroscopic transglenoid suture capsulobral repair: five-year followup. Am J Sports Med 1997;25:614-18.

93. Pap G, Machner A, Heitmann D, Merk H, Neumann HW. Recurrent luxation after arthroscopic refixation of the labrum using suture anchors in traumatic ventral shoulder luxation. Zentralb/ Chir 2001;126:199-204.

94. Kim SH, Ha KI, Kim YM. Arthroscopic revision Bankart repair: a prospective outcome study. Arthroscopy 2002;18:469-82.

95. Levine WN, Arroyo JS, Pollock RG, Flatow EL, Bigliani LU. Open revision stabilization surgery for recurrent anterior glenohumeral instability. Am J Sports Med 2000;28:156-60.

96. Lazarus MD, Harryman DT 2nd. Complications of open anterior stabilization of the shoulder. J Am Acad Orthop Surg 2000;8:122-32.

97. Lane JG, Sachs RA, Riehl B. Arthroscopic staple capsulorrhaphy: a long-term follow-up. Arthroscopy 1993;9:190-4.

98. Green MR, Christensen KP. Arthroscopic versus open Bankart procedures: a comparison of early morbidity and complication. Arthroscopy 1993:9:371-4.

99. Bohnsack M, Ruhmann O, Hurschler C, et al. Arthroscopic anterior shoulder stabilization: combined multiple suture repair and laser-assisted capsular shrinkage. Injury 2002;33:795-9

100. Kandziora F, Jager A, Bischof $\mathbf{F}$, et al. Arthroscopic labrum refixation for posttraumatic anterior shoulder instability: suture anchor versus transglenoid fixation technique. Arthroscopy 2000;16:359-66.

101.LandsiedI F. Arthroscopic therapy of recurrent anterior luxation of the shoulder by capsular repair. Arthroscopy 1992;8:296-304.

102. Matthews LS, Vetter WL, Oweida SJ, Spearman J, Helfet DL. Arthroscopic staple capsulorrhaphy for recurrent anterior shoulder instability. Arthroscopy 1988:4: 106-11.

103. Hawkins RB. Arthroscopic stapling repair for shoulder instability: a retrospective study of 50 cases. Arthroscopy 1989;5:122-8.

104.Ekelund A, Nyberg R. Arthroscopic shoulder stabilization using transglenoid sutures. Scand J Med Sci Sports 1998;8:416-20.

105.Segmuller HE, Hayes MG, Saies AD. Arthroscopic repair of glenolabral injuries with an absorbable fixation device. J Shoulder Elbow Surg 1997;6:383-92.

106. Karlsson J, Kartus J, Ejerhed L, et al. Bioabsorbable tacks for arthroscopic treatment of recurrent anterior shoulder dislocation. Scand J Med Sci Sports 1998;8: 411-15.

107. Kailes SB, Richmond JC. Arthroscopic vs. open Bankart reconstruction: a comparison using expected value decision analysis. Knee Surg Sports Traumatol Arthrosc 2001:9:379-85. 